\title{
Circular spectropolarimetric sensing of higher plant and algal chloroplast structural variations
}

\author{
C. H. Lucas Patty ${ }^{1}$ (D ) Freek Ariese ${ }^{2} \cdot$ Wybren Jan Buma $^{3} \cdot$ Inge Loes ten Kate $^{4} \cdot$ Rob J. M. van Spanning ${ }^{5} \cdot$ Frans Snik $^{6}$
}

Received: 7 June 2018 / Accepted: 4 August 2018 / Published online: 23 August 2018

(c) The Author(s) 2018

\begin{abstract}
Photosynthetic eukaryotes show a remarkable variability in photosynthesis, including large differences in light-harvesting proteins and pigment composition. In vivo circular spectropolarimetry enables us to probe the molecular architecture of photosynthesis in a non-invasive and non-destructive way and, as such, can offer a wealth of physiological and structural information. In the present study, we have measured the circular polarizance of several multicellular green, red, and brown algae and higher plants, which show large variations in circular spectropolarimetric signals with differences in both spectral shape and magnitude. Many of the algae display spectral characteristics not previously reported, indicating a larger variation in molecular organization than previously assumed. As the strengths of these signals vary by three orders of magnitude, these results also have important implications in terms of detectability for the use of circular polarization as a signature of life.
\end{abstract}

Keywords Circular polarization $\cdot$ Photosynthesis $\cdot$ Chloroplast $\cdot$ Chlorophyll $\cdot$ Algae

\section{Introduction}

Terrestrial biochemistry is based upon chiral molecules. In their most simple form, these molecules can occur in a left-handed and a right-handed version called enantiomers. Unlike abiotic systems, nature almost exclusively uses these molecules in only one configuration. Amino acids, for instance, primarily occur in the left-handed configuration while most sugars occur in the right-handed configuration. This exclusive use of one set of chiral molecules over the

C. H. Lucas Patty

lucas.patty@vu.nl

1 Molecular Cell Physiology, VU Amsterdam, De Boelelaan 1108, $1081 \mathrm{HZ}$ Amsterdam, The Netherlands

2 LaserLaB, VU Amsterdam, De Boelelaan 1083, 1081 HV Amsterdam, The Netherlands

3 HIMS, Photonics Group, University of Amsterdam, Science Park 904, 1098 XH Amsterdam, The Netherlands

4 Department of Earth Sciences, Utrecht University, Budapestlaan 4, 3584 CD Utrecht, The Netherlands

5 Systems Bioinformatics, VU Amsterdam, De Boelelaan 1108, 1081 HZ Amsterdam, The Netherlands

6 Leiden Observatory, Leiden University, P.O. Box 9513, 2300 RA Leiden, The Netherlands other, called homochirality, therefore serves as a unique and unambiguous biosignature (Schwieterman et al. 2018).

Many larger, more complex biomolecules and biomolecular architectures are chiral too and the structure and functioning of biological systems is largely determined by their chiral constituents. Homochirality is required for processes ranging from self-replication to enzymatic functioning and is therefore also deeply interwoven with the origins of life.

The phenomenon of chirality, i.e., the molecular dissymmetry of chiral molecules, causes a specific response to light (Fasman 2013; Patty et al. 2018a). This response is both dependent on the intrinsic chirality of the molecular building blocks and on the chirality of the supramolecular architecture. Polarization spectroscopy enables these molecular properties to be probed non-invasively from afar and is therefore of great value for astrobiology and the search for life outside our solar system. Polarization spectroscopy also has a long history in biological and chemical sciences. Circular dichroism (CD) spectroscopy utilizes the differential electronic absorption response of chiral molecules to leftand right-handed circularly polarized incident light and is very informative for structural and conformational molecular dynamics. As such it has proven to be an indispensable tool in (bio-)molecular research.

Chirality can also be observed in chlorophylls and bacteriochlorophylls utilized in photosynthesis. While their 
Fig. 1 Evolutionary relationships based on the host rRNA (left) and based on chloroplast DNA (cpDNA) (right)

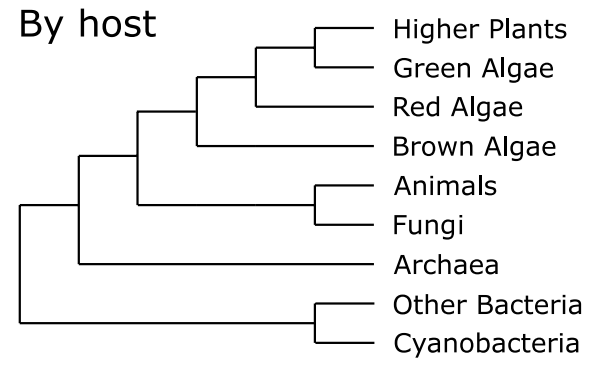

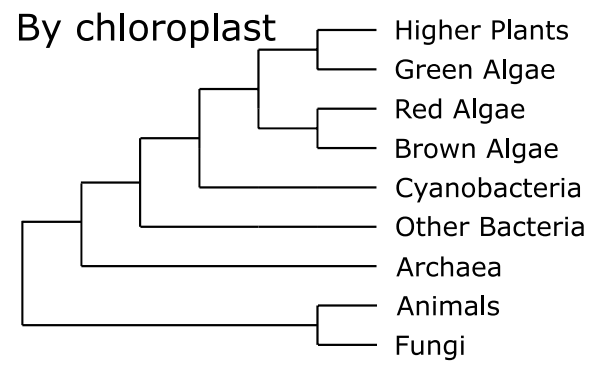

intrinsic CD signal is very weak due to their almost planar symmetrical structure, these chlorophylls are organized in a chiral supramolecular structure that greatly enhances these signals (Garab and van Amerongen 2009). This is particularly the case for the photosynthetic machinery in certain eukaryotes, where photosynthesis is carried out in specialized organelles, chloroplasts, which in higher plants have a large molecular density yielding anomalously large signals: polymer- and salt-induced (psi)-type circular dichroism (Keller and Bustamante 1986; Garab and van Amerongen 2009; Garab et al. 1991a; Tinoco et al. 1987).

While circular dichroism spectroscopy depends on the modulation of incident light to detect the differential extinction of circularly polarized light, we have recently shown that in leaves comparable results can be obtained by measuring the induced fractional circular polarization of unpolarized incident light (Patty et al. 2017, 2018b). As the latter only requires modulation in front of the detector it offers unique possibilities, allowing to probe the molecular architecture from afar. In vegetation, the influence of photosynthesis functioning and vegetation physiology on the polarizance could provide valuable information in Earth remote sensing applications, as was demonstrated for decaying leaves (Patty et al. 2017). As homochirality is a prerequisite for these signals (left- and right-handed molecules display an exactly opposite signal and will thus cancel out each other if present in equal numbers) and is unique to nature, circular polarization could also indicate the unambiguous presence of life beyond Earth and as such is a potentially very powerful biosignature (Sparks et al. 2009a, b; Wolstencroft 1974; Patty et al. 2018a; Pospergelis 1969; Schwieterman et al. 2018).

Higher plants evolved relatively recently in contrast to microbial life. Biosignatures of microbial life are mostly focused on astrobiology [and which also display typical circular polarization signals (Sparks et al. 2009a)]. While molecular analysis suggests higher plants appeared by 700 Ma (Heckman et al. 2001), the earliest fossil records date back to the middle Ordovician ( $\sim 70 \mathrm{Ma}$ ) (Wellman and Gray 2000). The earliest microbial fossil records date back to $3.7 \mathrm{Ga}$ (Nutman et al. 2016) and oxygenic photosynthesis (in cyanobacteria) is likely to have evolved before $2.95 \mathrm{Ga}$ (Planavsky et al. 2014). It is however unclear if photosynthetic microbial life would be able to colonize terrestrial niches extensively enough to be used as a remotely detectable biosignature.

On the other hand, these photosynthetic bacteria stood at the basis of the evolution of higher plants as their photosynthetic apparatus evolved from a endosymbiosis between a cyanobacterium and a heterotrophic host cell. It is widely accepted that all chloroplasts stem from a single primary endosymbiotic event (Moreira et al. 2000; Ponce-Toledo et al. 2017; McFadden 2001). Not all photosynthetic eukaryotes, however, descend from this endosymbiotic host, as certain algae acquired photosynthesis through secondary endosymbiosis of a photosynthetic eukaryote (McFadden 2001; Green 2011). The simplified evolutionary relations between the different algae, based on the host and on the chloroplasts, are shown in Fig. 1.

Although algae contribute up to $40 \%$ of the global photosynthesis (Andersen 1992), they have received limited attention in astrobiology so far. While not as ancient as microbial life, algae are considerably older than plants, with fossil evidence of red algae dating back to $1.6 \mathrm{Ga}$ (Bengtson et al. 2017). Additionally, molecular research on algae has mainly focused on a few unicellular algae, rather than multicellular species, and systematic studies on the chiral macro-organization of algal photosynthesis are lacking (Garab and van Amerongen 2009). Despite the common origin, millions of years of evolution has caused chloroplasts to show a remarkable diversity and flexibility in terms of structure (Fig. 2).

In higher plants, the chloroplasts typically display cylindrical grana stacks of 10-20 membrane layers that have a diameter of 300-600 $\mathrm{nm}$. The stacks are interconnected by lamellae of several hundred $\mathrm{nm}$ in length (Mustárdy and Garab 2003). Additionally, certain plants can display grana stacks of more than 100 membrane layers (Anderson et al. 1973, Steinmann and Sjöstrand 1955) while the bundle sheath cells of certain $\mathrm{C} 4$ plants, such as maize, lack stacked grana and only contain unstacked stroma lamellae (FaludiDaniel et al. 1973).

In higher plants, the psi-type circular polarizance is largely dependent on the size of the macrodomains formed by the photosystem II light-harvesting complex II supercomplexes (PSII-LHCII). The structure of PSII-LHCII in higher plants is relatively well known and consists of a dimeric 


\section{Higher Plants}

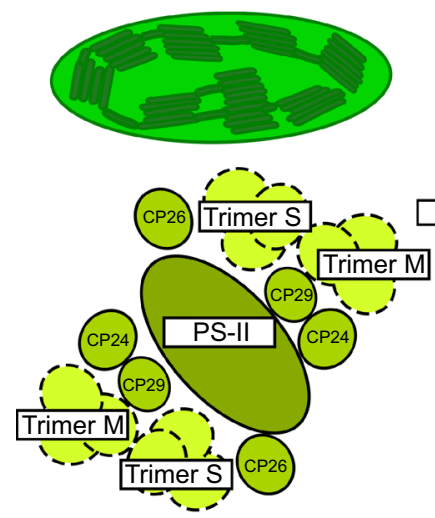

Red Algae

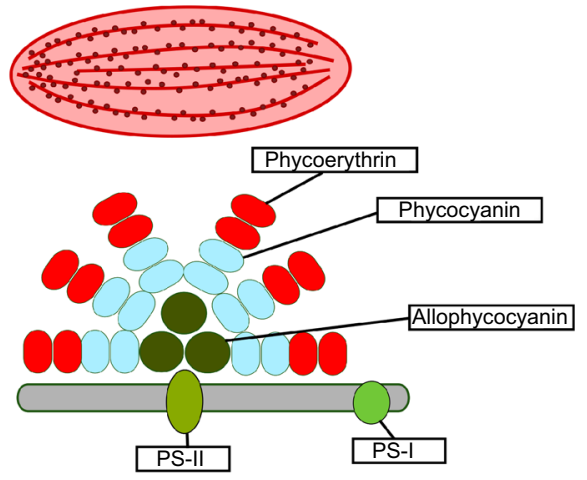

Fig. 2 Schematic representation of the photosynthetic structures of higher plants and algae. There is a distinct organizational difference in the supercomplexes between higher plants and algae. Additionally, while green algae display stacked thylakoid membranes, they lack

PSII core complex $\mathrm{C}_{2}$ and associated trimeric LHCII, subdivided in three types based on their position and association with the core: Loose (L), Moderate (M), and Strong (S). Additionally, three minor antennae occur as monomers (CP24, CP26, CP29) (Boekema et al. 1999). The position of trimer $\mathrm{L}$ is still unclear and has so far only been observed in spinach (Boekema et al. 1999). The protein constituents and their typical circular polarization signature have been determined by Tóth et al. (2016). Furthermore, the negative band of the psi-type split signal is associated with the stacking of the thylakoid membranes, whereas the positive band is associated with the lateral organization of the chiral domains (Garab et al. 1988a, 1991b; Cseh et al. 2000).

The evolutionary history of grana and their functional advantage has been a matter of debate. It has been proposed that the structural segregation by grana of PSII and PSI prevents excitation transfer between these systems (Albertsson 2001; Nevo et al. 2012; Trissl and Wilhelm 1993). The

\section{Green Algae}
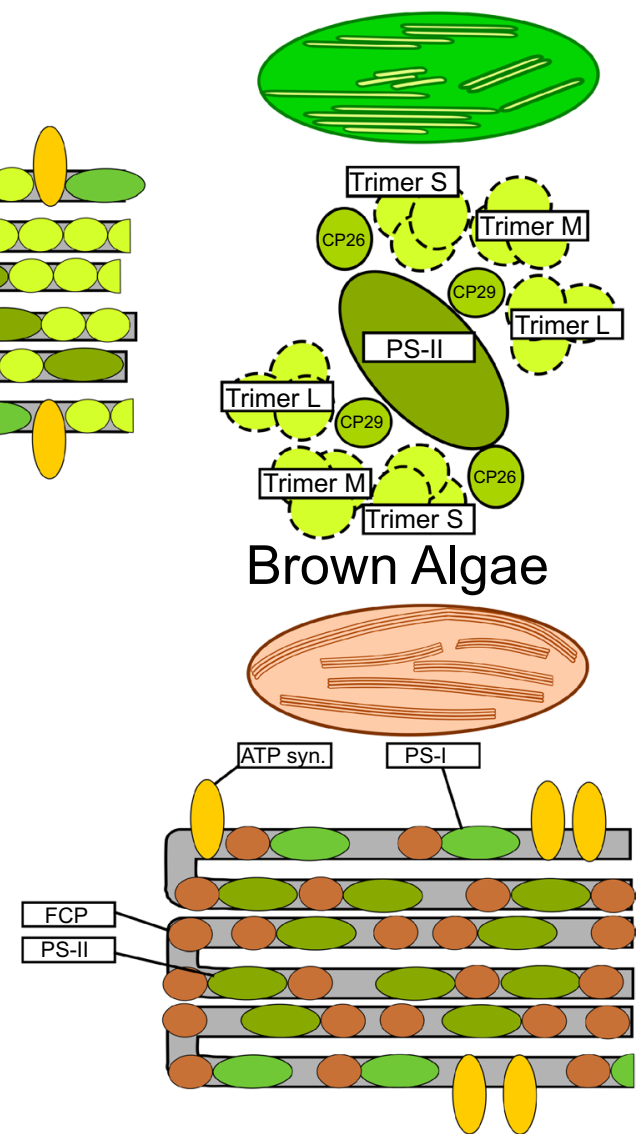

true grana. Red algae contain phycobilisomes, unlike the other algae. In brown algae the thylakoid membranes are threefold and the supercomplex organization is not entirely resolved

extended compartmentation brought upon by grana might also aid regulatory pathways such as used in carbon fixation (Anderson 1999). It has been suggested that grana facilitates the regulation of light harvesting and enhance PSII functioning from limiting to saturating light levels, while at the same time protecting it from sustained high irradiance (Anderson 1999). Together with other adaptations, it has been hypothesized that these changes might have ultimately enabled green algae/plants to colonize and dominate various terrestrial niches (Nevo et al. 2012). Others have suggested that it might simply be a lack of competition; red algae for instance have probably experienced several evolutionary bottlenecks, vastly decreasing their genome size and therewith their potential for evolutionary adaptation (Collen et al. 2013).

Most closely related to higher plants are the green algae, which share a quite recent common ancestor. Similar to higher plants, green algae contain chlorophyll $a$ and $b$. The 
structural composition of their photosynthetic machinery and the associated genes is primarily known from the unicellular green algae Chlamydomonas. Despite the high sequence similarity there are significant differences between the supercomplexes of higher plants and green algae. Importantly, green algae lack CP24, resulting in a different organization of the PSII-LHCII supercomplex (Tokutsu et al. 2012). While many green algae display thylakoid stacking, which can be up to seven membrane layers thick (Remias et al. 2005), true grana in green algae are rare and only occur in the late branching taxa Coleochaetales and Charales (Gunning and Schwartz 1999; Larkum and Vesk 2003).

Red algae also contain thylakoid membranes but these are never stacked. Furthermore, unlike green algae and plants, red algae can contain chlorophyll $d$, a pigment with an absorption band from 700 to $730 \mathrm{~nm}$ (Larkum and Kühl 2005). The red algae also contain phycobilisomes that serve as the primary antennae for PSII rather than the chlorophyll binding proteins found in higher plants and other algae. These phycobilisomes are homologous to those in cyanobacteria, but are lacking in plants and other algae (McFadden 2001).

Similarly, brown algae do not possess stacked thylakoid membranes but also do not contain phycobilins. All brown algae contain chlorophyll $a$ and usually chlorophyll $\mathrm{C}_{1}, \mathrm{C}_{2}$, and/or $\mathrm{C}_{3}$. The light-harvesting systems in brown algae are based on fucoxanthin chlorophyll $\mathrm{a} / \mathrm{c}_{\{1,2,3\}}$ proteins (FCP), which are homologous to LHC in higher plants/green algae but have a different pigment composition and organization (Premvardhan et al. 2010; Büchel 2015). Although this is still under debate (Burki et al. 2016), the brown algae have been classified as one supergroup (Dorrell and Smith 2011). Most brown algae have chloroplasts which were acquired through one or more endosymbiotic events with red algae (Dorrell and Smith 2011). Additionally, certain species of brown algae have been shown to display psi-type circular polarizance, although varying magnitudes of these signals have been reported, ranging from very weak to signals similar to higher plants [see (Garab and van Amerongen 2009) and references therein].

In the present study, we measure the fractional circular polarizance of various higher plants and multicellular algae. As the level of chiral macro-organization varies greatly between unicellular algae, we expect especially in multicellular algae that the organization can reach a higher or different level of complexity. These studies will additionally assess the feasibility of biosignature detection for (eukaryotic) photosynthesis from different evolutionary stages. While transmission and reflectance generally show a comparable spectral profile, the signals in reflectance are often weaker (e.g., due to surface glint). In the present study, we will therefore only display the results in transmission, as it provides better sensitivity for small spectral changes between samples.

\section{Materials and methods}

\section{Sample collection}

Ulva lactuca, Porphyra sp., and Saccharina latissima were grown in April at the Royal Netherlands Institute for Sea Research (NIOZ), using natural light and seawater. The algae were transported and stored in seawater at room temperature. Measurements on the algae were carried out within 2 days after acquisition.

Ulva sp., Undaria pinnatifida, Grateloupia turuturu, $S$. latissima, Fucus serratus, and Fucus spiralis were collected by Guido Krijger from WildWier ${ }^{1}$ from the North Sea near Middelburg in February. The algae were transported under refrigeration and stored in seawater. Measurements on the algae were carried out within 2 days after acquisition.

Leaves of Skimmia japonica and Prunus laurocerasus were collected in January from a private backyard garden near the city center of Amsterdam, Aspidistra elatior was obtained from the Hortus Botanicus Vrije Universiteit Amsterdam in February.

\section{Spectropolarimetry}

For all measurements, three different samples were used $(n=3)$ and each single measurement is the average of at least 20,000 repetitions. Before each measurement, the samples were padded with paper towels to remove excess surface water. Circular polarization measurements were carried out in transmission and were performed using TreePol. TreePol is a dedicated spectropolarimetric instrument developed by the Astronomical Instrumentation Group at the Leiden Observatory (Leiden University). The instrument was specifically developed to measure the fractional circular polarization (V/I) of a sample interacting with unpolarized light as a function of wavelength (400-900 nm) and is capable of fast measurements with a sensitivity of $\sim 1 \times 10^{-4}$. TreePol applies spectral multiplexing with the implementation of a dual fiber-fed spectrometer using ferro-liquid-crystal (FLC) modulation synchronized with fast read-out of the one-dimensional detector in each spectrograph, in combination with a dual-beam approach in which a polarizing beam splitter feeds the two spectrographs with orthogonally polarized light [see also (Patty et al. 2017)].

\footnotetext{
1 Any mention of commercial products or companies within this paper is for information only; it does not imply recommendation or endorsement by the authors or their affiliated institutions.
} 
Fig. 3 Circular polarimetric spectra of S. japonica, P. laurocerasus, and $A$. elatior leaves. Shaded areas denote the standard error, $n=3$ per species
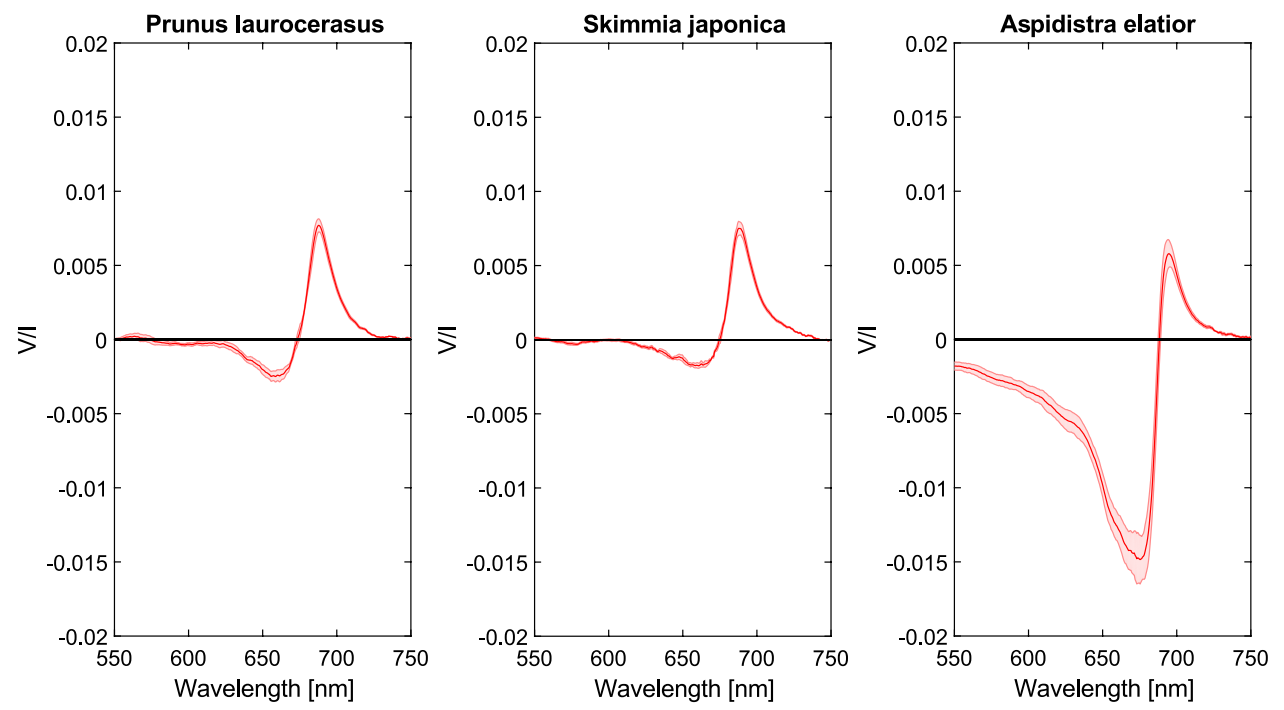

In this study, we have measured the induced fractional circular polarizance normalized by the total transmitted light intensity $(V / I)$. Circular dichroism measures the differential absorption of left- or right-handed circularly polarized incident light, which is often reported in degrees $\theta$. Under certain conditions, these two can be related and can therefore be converted by $V / I \approx \frac{2 \pi \theta_{\text {deg }}}{180}$ [see also (Patty et al. 2018a)]. It has been shown that for leaves in transmission, the induced polarizance and the differential absorbance are comparable (Patty et al. 2017; 2018b), but we have not verified this for the samples used in this study.

\section{Results}

\section{Higher plants}

The circular polarization spectra of three different higher plants are shown in Fig. 3. For all species, we observe the typical split signal around the chlorophyll $a$ absorption band $(\approx 680 \mathrm{~nm})$ with a negative band at $\approx 660 \mathrm{~nm}$ and a positive band at $\approx 690 \mathrm{~nm}$. The spectra of Skimmia and Prunus are very similar to each other in both shape and magnitude and show no significant differences. These results are also very similar to the results obtained for most other higher plants (data not shown). Interestingly, the circular polarimetric spectrum of A. elatior shows an exceedingly large negative band $\left(-1.5 \times 10^{-2}\right)$ with a noticeable negative circular polarization extending much further into the blue, beyond the chlorophyll $a$ (but also $b$ ) absorption bands. The positive band, however, has a similar magnitude $\left(+6 \times 10^{-3}\right)$ as the other two plant species.

\section{Green algae}

The circular polarization spectra of two different green algae are shown in Fig. 4. Similar to higher plants, a split signal is observed around the chlorophyll $a$ absorption band $(\approx 680 \mathrm{~nm})$. Unlike higher plants, however, the negative and positive bands do not seem to overlap. The negative band reaches a $V / I$ minimum at $\approx 655 \mathrm{~nm}$ and the positive band reaches a maximum at $\approx 690 \mathrm{~nm}$, but the $V / I$ signal is close to 0 , and thus shows no net circular polarization between $\approx 665$ to $678 \mathrm{~nm}$. Additionally, the magnitude of the signals is much smaller than that of higher plants.
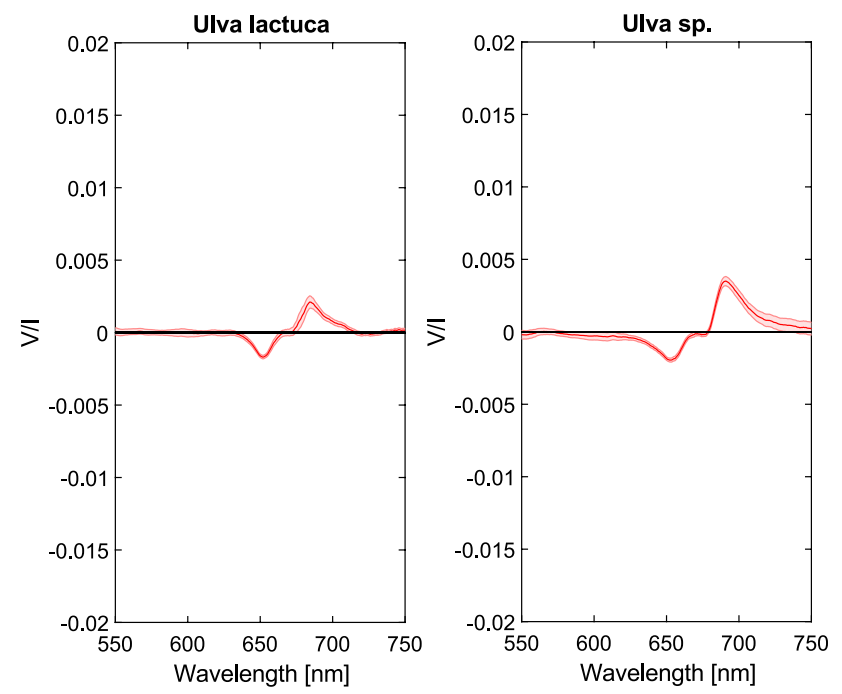

Fig. 4 Circular polarimetric spectra of U. lactuca and Ulva sp. green algae. Shaded areas denote the standard error, $n=3$ per species 

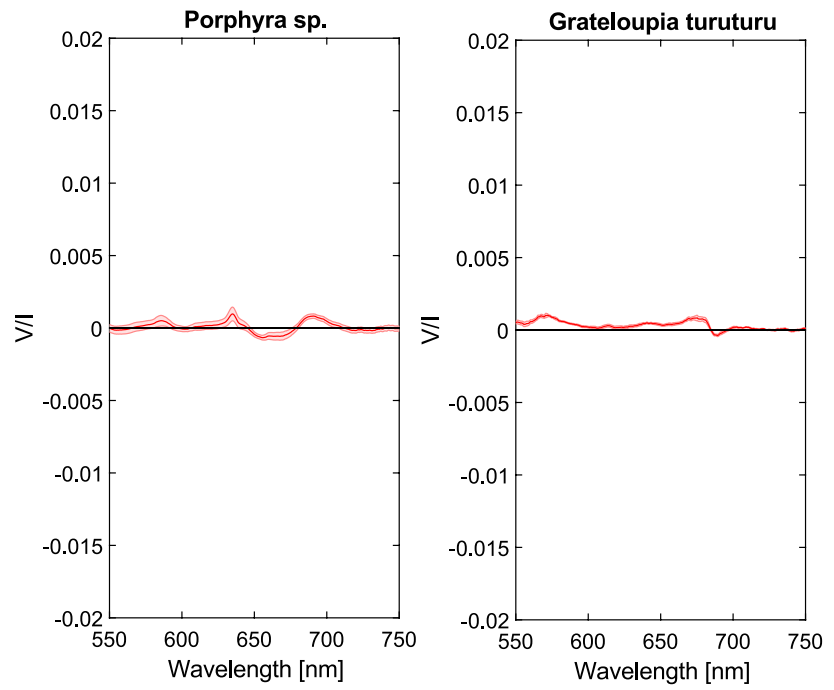

Fig. 5 Circular polarimetric spectra of Porphyra sp. and G. turuturu red algae. Shaded areas denote the standard error, $n=3$ per species

\section{Red algae}

We show the circular polarization spectra of two different red algae in Fig. 5. These spectra show distinct differences compared to the higher plants and the green or brown algae. Porphyra sp. shows a continuous split signal around $\approx 680 \mathrm{~nm}$, and an additional sharp positive feature at $\approx 635 \mathrm{~nm}$. G. turuturu lacks these features and shows an inverse split signal around $\approx 680 \mathrm{~nm}$. In both species, non-zero circular polarization can also be observed between 550 and $600 \mathrm{~nm}$. We will further interpret these results in the Discussion.

\section{Brown algae}

The brown algae exhibit a lot of variation in signal strength. For ease of comparison, the results of our circular spectropolarimetric measurements are plotted in Figs. 6 and 7 on the same y-scale. Figure 6 makes clear that a juvenile $S$. latissima barely displays a significant signal with the exception of a very weak negative feature $\left(V / I=-4 \times 10^{-4}\right)$. The mature $S$. latissima samples show somewhat stronger bands, although the signal is still relatively small $\left(-1 \times 10^{-3}\right.$, $+1 \times 10^{-3}$ ). The polarimetric spectra of the brown algae $U$. pinnatifida, display a larger signal comparable to that of higher vegetation.

Interestingly, the polarimetric spectra of the brown algae of the genus Fucus display very large circular polarization signals, see Fig. 7. The alga Fucus spiralis has a V/I minimum and maximum of $-8 \times 10^{-3}$ and $+2 \times 10^{-2}$, respectively. Additionally, the bands are relatively narrow, with less polarization outside the chlorophyll $a$ absorbance band. In the polarimetric spectra of $F$. spiralis, and to a lesser extent also of $U$. pinnatifida, a small negative band can be observed at $720 \mathrm{~nm}$. Additionally, in the spectra of both $F$. serratus and $F$. spiralis, a positive band can be observed at $595 \mathrm{~nm}$.

\section{V/I versus absorbance}

The V/I maxima and minima versus the absorbance are shown in Fig. 8. A slight correlation is visible between the maximum and minimum magnitude of the $V / I$ bands within $650 \mathrm{~nm}$ to $700 \mathrm{~nm}$ and the absorbance over $675 \mathrm{~nm}$ to 685 $\mathrm{nm}$. In general, the magnitude of the bands increases with increasing absorbance. Both $F$. serratus and F. spiralis show positive and negative bands with a very large magnitude
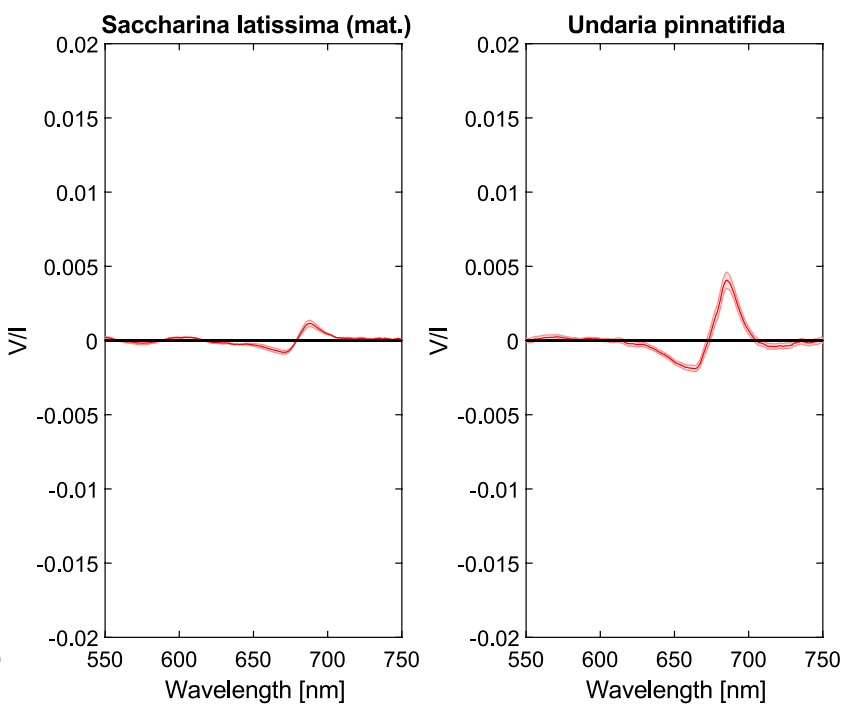

Fig. 6 Circular polarimetric spectra of S. latissima (juvenile and mature) and $U$. pinnatifida brown algae. Shaded areas denote the standard error, $n=3$ per species

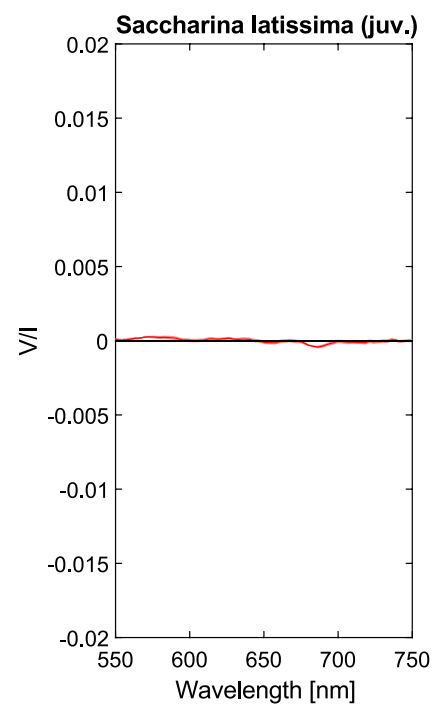
. 

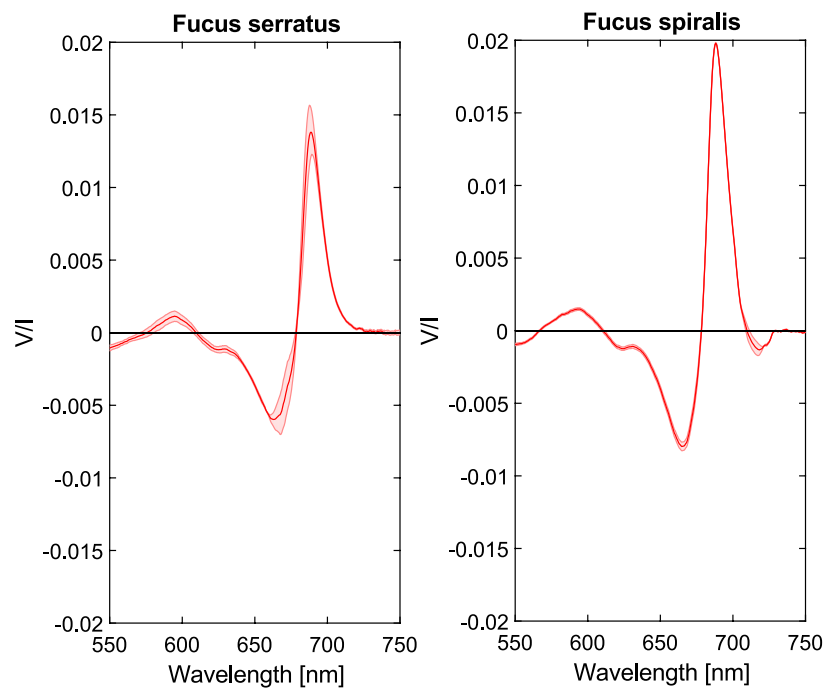

Fig. 7 Circular polarimetric spectra of $F$. serratus and $F$. spiralis brown algae. Shaded areas denote the standard error, $n=3$ per species

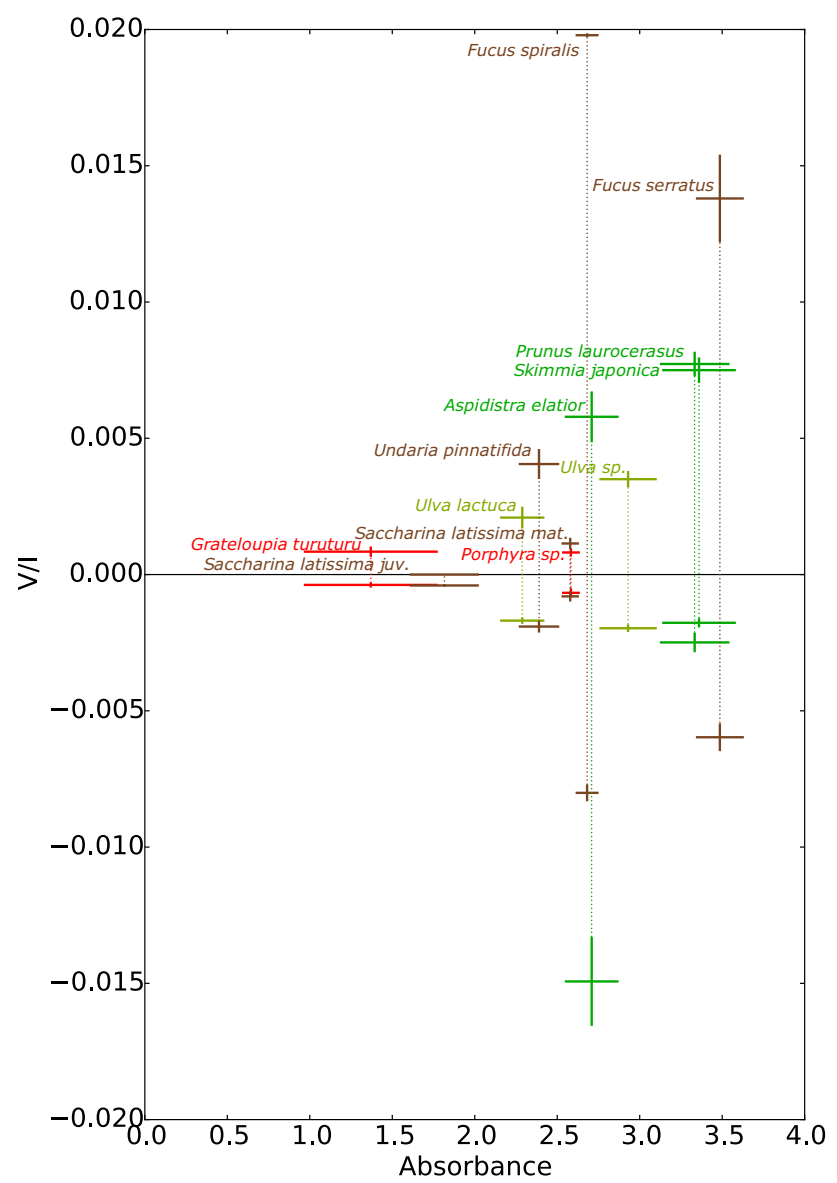

Fig. 8 Maximum extend of the $V / I$ bands within $650 \mathrm{~nm}$ to $700 \mathrm{~nm}$ against the absorbance over $675 \mathrm{~nm}$ to $685 \mathrm{~nm}$. Error bars denote the standard error for $n=3$ per species

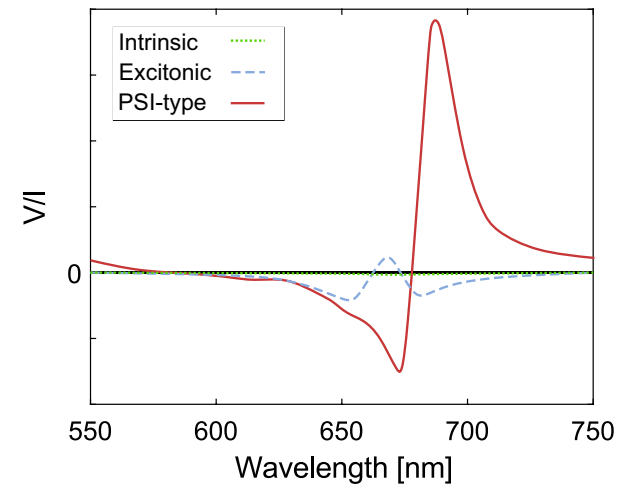

Fig. 9 The three major sources of circular polarizance around the chlorophyll absorbance band in the red for higher plants for identical chlorophyll concentrations. Adapted after (Garab and van Amerongen 2009)

well outside this trend. This is similar for the large negative band of A. elatior. On the other hand, mature S. latissima and Porphyra sp. have a relatively low circular polarizance.

\section{Discussion}

Different eukaryotic phototrophic organisms display different circular polarization spectra, with signal magnitudes that can vary by two orders of magnitude. Chlorophyll $a$ itself exhibits a very weak intrinsic circular polarizance around $680 \mathrm{~nm}$ (Garab and van Amerongen 2009). Excitonic coupling between chlorophylls leads to a much larger signal in phototrophic bacteria and certain algae. In many more developed phototrophic organisms, the polarization spectra are dominated by the density and handedness of the supramolecular structures (psi-type circular dichroism), although these signals are superimposed on each other. Thus, for identical chlorophyll concentrations, the polarimetric spectral characteristics can vastly differ depending on the organization (see also Fig. 9).

The typical psi-type circular spectropolarimetric signals observed in vegetation are the result of the superposition of two relatively independent signals resulting from different chiral macrodomains in the chloroplast (Garab et al. 1988b, c, 1991a; Finzi et al. 1989). These psi-type bands of opposite sign do not have the same spectral shape and thus do not cancel each other out completely. The negative band is predominantly associated with the stacking of the thylakoid membranes, whereas the positive band mainly derives from the lateral organization of the chiral macrodomains formed by the PSII-LHCII complexes (Cseh et al. 2000; Dobrikova et al. 2003; Jajoo et al. 2012; Garab et al. 1991a).

Plant chloroplasts generally show little variation in structure (Staehelin 1986), which is noticeable in the circular polarization spectra of most plants (e.g., see the spectra of 
Skimmia and Prunus in Fig. 3). It has been reported before that the cpDNA sequences are extraordinarily conserved among plants and nearly identical in ferns, gymnosperms, and angiosperms (Palmer and Stein 1986). Of course, certain plants contain more chloroplasts per cell, or contain chloroplasts which are significantly larger or smaller, but in both cases, the normalized circular polarization will remain the same.

The polarimetric spectra of Aspidistra (Fig. 3) show a remarkably intense negative band, unlike the results usually encountered in plants. The positive band, however, has a magnitude that can be expected based on the lower absorbance as compared to the other higher plants we measured (see also Fig. 8). It has been shown that the contribution of both the negative and the positive band is dependent on the alignment of the chloroplasts (Garab et al. 1988c, 1991a), which might locally be aligned in such a way that only a single band dominates [e.g., near the veins of leaves (Patty et al. 2018b)]. The polarimetric spectra of Aspidistra, however, can be very well explained by the unusually large grana. Previous electron microscopy research on Aspidistra elatior chloroplasts revealed grana containing a vast number of thylakoid layers that may well exceed 100 (Steinmann and Sjöstrand 1955). As the positive and the negative bands overlap (leading to the split signal), it is to be expected that also the positive band is larger than encountered normally.

Similar to higher plants, also green algae contain PSII-LHCII supercomplexes utilized in photosynthesis. Between green algae and higher plants there are slight differences in the trimeric LHCII proteins and their isoforms, and, in addition, the green algae lack one of three minor monomeric LHCII polypeptides (CP24) [see also (Minagawa 2013) and references therein]. The green algae we measured show a spectral polarimetric profile that appears very similar to that of plants. However, the negative band centered around $650 \mathrm{~nm}$ is likely an excitonic band resulting from short-range interactions of the chlorophylls and the negative, usually stronger, psi-type band around $675 \mathrm{~nm}$ is virtually absent. The positive psi-type centered around 690 $\mathrm{nm}$, on the other hand, is still present.

These results are unlike those reported for the unicellular green algae Chlamydomonas reinhardtii, which display a negative excitonic and a negative psi-type band of equal strength [e.g., see (Nagy et al. 2014)]. Importantly, the PSII-LHCII supercomplexes are far less stable in green algae as compared to plants, and it has been indicated that the $\mathrm{L}$ trimer (as well as the $\mathrm{M}$ and $\mathrm{S}$ trimers) could dissociate easily from PSII (Tokutsu et al. 2012). It has been shown that in Ulva flattening of the chloroplasts occurs under illumination, which additionally results in a decrease in thickness of the thylakoid membrane itself (Murakami and Packer 1970). Such fundamental changes in molecular structure might easily lead to (partial) dissociation of trimer
L, which in turn can lead to the observed apparent absence of the negative psi-type band.

The red algae contain a more primitive photosynthetic apparatus that represents a transition between cyanobacteria and the chloroplasts of other algae and plants. This is also very evident from the displayed spectra in Fig. 5. For both species, the magnitude of the signal is small and comparable, even though Porphyra sp. had a much larger absorbance (Fig. 8), but the spectral shape suggests very fundamental differences in molecular structure. Surprisingly, Porphyra sp. shows a circular polarization spectrum with bands that might be associated with psi-type circular polarizance [at $675 \mathrm{~nm}(-)$ and at $690 \mathrm{~nm}(+)]$. The origin and significance of these signals, however, require further investigation. The circular polarimetric spectra of $G$. turuturu lack these features but show two bands that can be associated with the excitonic circular polarization bands similar to those in cyanobacteria [at $670 \mathrm{~nm}(+)$ and at $685 \mathrm{~nm}(-)$ ] [cf. (Sparks et al. 2009a)], which for a large part result from the excitonic interactions in PSI (Schlodder et al. 2007). In both species, the features between 550 and $600 \mathrm{~nm}$ might be associated with R-phycoerythrin (Bekasova et al. 2013). Additionally, in Porphyra sp., the sharp feature around $635 \mathrm{~nm}$ can be associated with phycocyanin (Sparks et al. 2009a). Both pigment-protein complexes belong to the phycobilisomes, which only occur in red algae and cyanobacteria and function as light-harvesting antennae for PSII while LHC is limited to PSI.

As in red algae and green algae, the brown algae contain no true grana but the thylakoid membranes are stacked in groups of three (Berkaloff et al. 1983). The brown algae measured in this study additionally contain chlorophyll $c$, which is slightly blue-shifted compared to chlorophyll $a$ or $b$. Compared to chlorophyll $a$, chlorophyll $c$, however, has only a very weak contribution to the overall circular polarizance. Additionally, in brown algae, the light-harvesting antennae are homogeneously distributed along the thylakoid membranes (De Martino et al. 2000; Büchel and Garab 1997).

Interestingly, the juvenile Saccharina displays only a very weak negative band around $683 \mathrm{~nm}$ (Fig. 6). These results closely resemble those of isolated brown algae LHCs, which exhibit no excitonic bands but show solely a negative band around $680 \mathrm{~nm}$. This band likely results from an intrinsic induced chirality of the chlorophyll $a$ protein complex (Büchel and Garab 1997). The polarimetric spectra of mature Saccharina and Undaria show a split signal that is similar to that of higher plants. While the molecular architecture of the LHCs is very different from those in higher plants, the pigment-protein complexes in brown algae are organized in large chiral domains which give similar psi-type signals in circular polarizance (Szabó et al. 2008; Nagy et al. 2012). These intrinsic so-called fucoxanthin chlorophyll $a / c$ binding 
proteins show a high homology to LHC in higher plants and have been shown to form complexes with trimers or higher oligomers (Lepetit et al. 2007; Büchel 2003; Katoh et al. 1989).

As shown in Fig. 7, the measured species of the genus Fucus exhibit an unusually large signal in circular polarizance, while the absorbance of the samples was within the range of the samples of the other species (Fig. 8). Although their spectral shapes are very similar to those of diatoms [cf. (Ghazaryan et al. 2016; Szabó et al. 2008; Büchel and Garab 1997)], the bands are two orders of magnitude stronger in Fucus. Most research on chlorophyll $a / c$ photosynthesis is, however, carried out on diatoms and the reported size of the protein complexes again varies. Signals of such magnitude suggest that these macromolecular assemblies are much larger in Fucus than previously reported for other brown algae. Additionally, in the spectra of Fucus, a positive band can be observed around $595 \mathrm{~nm}$. Most likely, this band and the weaker negative band around $625 \mathrm{~nm}$ can be assigned to chlorophyll $c$.

The results here show that the molecular and macromolecular organization of the photosynthetic machinery in algae is much more flexible and dynamic than reported before, likely due to larger inter-specific differences than generally assumed. Additionally, this also appears to be the case for one of the plants we measured (A. elatior), which displayed a negative psi-band one order of magnitude larger than ordinarily observed for higher plants.

When it comes to circular polarizance as a biosignature, it is important to note that efficient photosynthesis is not necessarily accompanied by large signals in circular polarization. While the intrinsic circular polarizance of chlorophyll is very low, the magnitude of the signals becomes greatly enhanced by a larger organization resulting in excitonic circular polarizance and ultimately psi-type circular polarizance. For the latter, the chiral organization of the macrodomains of the pigment-protein complexes is of importance, but it should be noted that the density of the complexes needs to be large enough (that is, significant coupling over the macrodomain is required) in order to function as a chiral macrodomain (Keller and Bustamante 1986). Many organisms thus display only excitonic circular polarizance, as is the case for certain algae measured in this study and generally bacteria. When psi-type circular polarizance is possible, the signals can easily become very large, in our study up to $2 \%$ for brown algae in transmission.

\section{Conclusions}

We have measured the polarizance of various multicellular algae representing different evolutionary stages of eukaryotic photosynthesis. We have shown that the chiral organization of the macrodomains can vary greatly between these species. Future studies using molecular techniques to further characterize and isolate the complexes in these organisms are highly recommended. It will additionally prove very interesting to investigate these chloroplasts (including those with larger grana such as Aspidistra) using polarization microscopy (e.g., Steinbach et al. 2014; Finzi et al. 1989; Gombos et al. 2008). The high-quality spectra in this study and their reproducibility underline the possibility of utilizing polarization spectroscopy as a quantitative tool for non-destructively probing the molecular architecture in vivo.

Our results not only show variations in spectral shapes, but also in magnitude. Especially, the brown algae show a large variation, which is up to three orders of magnitude for the species measured in this study. Additionally, the induced fractional circular polarization by members of the genus Fucus is much larger than observed in vegetation. Future studies on the supramolecular organization in this genus and the variability caused by, for instance, light conditions, will also clarify the maximum extent of the circular polarizance by oxygenic photosynthetic organisms.

While the displayed results were obtained in transmission, the spectral features are also present in reflection. As such, future use of circular spectropolarimetry in satellite or airborne remote sensing could not only aid in detecting the presence of floating multicellular algae but also aid in species differentiation, which is important in regional biogeochemistry (Dierssen et al. 2015).

Importantly, while the presence of similar circular polarization signals is an unambiguous indicator for the presence of life, life might also flourish on a planetary surface and still show minimal circular polarizance (which for instance would have been the case on Earth if terrestrial vegetation evolved through different Archaeplastida/SAR supergroup lineages). On the other hand, these signals might also be much larger than we would observe from an Earth disk-averaged spectrum (which is the unresolved and therefore spatially integrated spectrum of a planet).

Acknowledgements We thank Klaas Timmermans (NIOZ) and Guido Krijger (Wildwier) for providing us with the algae samples. We thank the Hortus Botanicus Vrije Universiteit Amsterdam for providing us with the Aspidistra samples. This work was supported by the Planetary and Exoplanetary Science Programme (PEPSci), Grant 648.001.004, of the Netherlands Organisation for Scientific Research (NWO).

Open Access This article is distributed under the terms of the Creative Commons Attribution 4.0 International License (http://creativeco mmons.org/licenses/by/4.0/), which permits unrestricted use, distribution, and reproduction in any medium, provided you give appropriate credit to the original author(s) and the source, provide a link to the Creative Commons license, and indicate if changes were made. 


\section{References}

Albertsson P-A (2001) A quantitative model of the domain structure of the photosynthetic membrane. Trends Plant Sci 6(8):349-354

Andersen R (1992) Diversity of eukaryotic algae. Biodivers Conserv 1(4):267-292

Anderson JM (1999) Insights into the consequences of grana stacking of thylakoid membranes in vascular plants: a personal perspective. Funct Plant Biol 26(7):625-639

Anderson J M, Goodchild D, Boardman N (1973) Composition of the photosystems and chloroplast structure in extreme shade plants. Biochim Biophys Acta 325:573-585

Bekasova O, Shubin V, Safenkova I, Kovalyov L, Kurganov B (2013) Structural changes in R-phycoerythrin upon CdS quantum dot synthesis in tunnel cavities of protein molecules. Int J Biol Macromol 62:623-628

Bengtson S, Sallstedt T, Belivanova V, Whitehouse M (2017) Threedimensional preservation of cellular and subcellular structures suggests 1.6 billion-year-old crown-group red algae. PLoS Biol 15(3):e2000735

Berkaloff C, Duval J, Hauswirth N, Rousseau B (1983) Freeze fracture study of thylakoids of Fucus serratus. J Phycol 19(1):96-100

Boekema EJ, van Roon H, van Breemen JFL, Dekker JP (1999) Supramolecular organization of photosystem II and its light-harvesting antenna in partially solubilized photosystem II membranes. Eur J Biochem 266(2):444-452

Burki F, Kaplan M, Tikhonenkov DV, Zlatogursky V, Minh BQ, Radaykina LV, Smirnov A, Mylnikov AP, Keeling PJ (2016) Untangling the early diversification of eukaryotes: a phylogenomic study of the evolutionary origins of Centrohelida, Haptophyta and Cryptista. In: Proc. R. Soc. B, vol. 283, p. 20152802, The Royal Society

Büchel C (2003) Fucoxanthin-chlorophyll proteins in diatoms: 18 and $19 \mathrm{kDa}$ subunits assemble into different oligomeric states. Biochemistry 42(44):13027-13034

Büchel C (2015) Evolution and function of light harvesting proteins. J Plant Physiol 172:62-75

Büchel C, Garab G (1997) Organization of the pigment molecules in the chlorophyll a/c light-harvesting complex of Pleurochloris meiringensis (Xanthophyceae). characterization with circular dichroism and absorbance spectroscopy. J Photochem Photobiol B 37(1-2):118-124

Collen J, Porcel B, Carre W, Ball SG, Chaparro C, Tonon T, Barbeyron T, Michel G, Noel B, Valentin K, Elias M, Artiguenave F, Arun A, Aury J-M, Barbosa-Neto JF, Bothwell JH, Bouget F-Y, Brillet L, Cabello-Hurtado F, Capella-Gutierrez S, Charrier B, Cladiere L, Cock JM, Coelho SM, Colleoni C, Czjzek M, Silva CD, Delage L, Denoeud F, Deschamps P, Dittami SM, Gabaldon T, Gachon CMM, Groisillier A, Herve C, Jabbari K, Katinka M, Kloareg B, Kowalczyk N, Labadie K, Leblanc C, Lopez PJ, McLachlan DH, Meslet-Cladiere L, Moustafa A, Nehr Z, Collen PN, Panaud O, Partensky F, Poulain J, Rensing SA, Rousvoal S, Samson G, Symeonidi A, Weissenbach J, Zambounis A, Wincker P, Boyen C (2013) Genome structure and metabolic features in the red seaweed Chondrus crispus shed light on evolution of the archaeplastida. Proce Natl Acad Sci USA 110(13):5247-5252

Cseh Z, Rajagopal S, Tsonev T, Busheva M, Papp E, Garab G (2000) Thermooptic effect in chloroplast thylakoid membranes. Thermal and light stability of pigment arrays with different levels of structural complexity. Biochemistry 39(49):15250-15257

De Martino A, Douady D, Quinet-Szely M, Rousseau B, Crépineau F, Apt K, Caron L (2000) The light-harvesting antenna of brown algae. FEBS J 267(17):5540-5549

Dierssen H, Chlus A, Russell B (2015) Hyperspectral discrimination of floating mats of seagrass wrack and the macroalgae
Sargassum in coastal waters of Greater Florida Bay using airborne remote sensing. Remote Sens Environ 167:247-258

Dobrikova A G, Várkonyi Z, Krumova S B, Kovács L, Kostov G K, Todinova S J, Busheva M C, Taneva S G, Garab G (2003) Structural rearrangements in chloroplast thylakoid membranes revealed by differential scanning calorimetry and circular dichroism spectroscopy. thermo-optic effect. Biochemistry 42:11272-11280

Dorrell RG, Smith AG (2011) Do red and green make brown?: perspectives on plastid acquisitions within chromalveolates. Eukaryot Cell 10(7):856-868

Faludi-Daniel A, Demeter S, Garay AS (1973) Circular dichroism spectra of granal and agranal chloroplasts of maize. Plant Physiol 52(1):54-56

Fasman GD (2013) Circular dichroism and the conformational analysis of biomolecules. Springer, New York

Finzi L, Bustamante C, Garab G, Juang C-B (1989) Direct observation of large chiral domains in chloroplast thylakoid membranes by differential polarization microscopy. Proc Natl Acad Sci USA 86(22):8748-8752

Garab G, van Amerongen H (2009) Linear dichroism and circular dichroism in photosynthesis research. Photosynth Res 101(2-3):135-146

Garab G, Faludi-Daniel A, Sutherland JC, Hind G (1988) Macroorganization of chlorophyll $\mathrm{a} / \mathrm{b}$ light-harvesting complex in thylakoids and aggregates: information from circular differential scattering. Biochemistry 27(7):2425-2430

Garab G, Finzi L, Bustamante C (1991) Differential polarization imaging of chloroplasts: microscopic and macroscopic linear and circular dichroism. Light Biol Med 2:271

Garab G, Kieleczawa J, Sutherland JC, Bustamante C, Hind G (1991) Organization of pigment-protein complexes into macrodomains in the thylakoid membranes of wild-type and chlorophyll fo-less mutant of barley as revealed by circular dichroism. Photochem Photobiol 54(2):273-281

Garab G, Leegood RC, Walker DA, Sutherland JC, Hind G (1988) Reversible changes in macroorganization of the light-harvesting chlorophyll a/b pigment-protein complex detected by circular dichroism. Biochemistry 27(7):2430-2434

Garab G, Wells S, Finzi L, Bustamante C (1988) Helically organized macroaggregates of pigment-protein complexes in chloroplasts: evidence from circular intensity differential scattering. Biochemistry 27(16):5839-5843

Ghazaryan A, Akhtar P, Garab G, Lambrev P H, Büchel C (2016) Involvement of the Lhcx protein Fcp6 of the diatom Cyclotella meneghiniana in the macro-organisation and structural flexibility of thylakoid membranes. Biochim Biophys Acta 1857(9):1373-1379

Gombos I, Steinbach G, Pomozi I, Balogh A, Vámosi G, Gansen A, László G, Garab G, Matkó J (2008) Some new faces of membrane microdomains: a complex confocal fluorescence, differential polarization, and fcs imaging study on live immune cells. Cytometry Part A 73(3):220-229

Green BR (2011) Chloroplast genomes of photosynthetic eukaryotes. Plant J 66(1):34-44

Gunning B, Schwartz O (1999) Confocal microscopy of thylakoid autofluorescence in relation to origin of grana and phylogeny in the green algae. Funct Plant Biol 26(7):695-708

Heckman DS, Geiser DM, Eidell BR, Stauffer RL, Kardos NL, Hedges SB (2001) Molecular evidence for the early colonization of land by fungi and plants. Science 293(5532):1129-1133

Jajoo A, Szabó M, Zsiros O, Garab G (2012) Low pH induced structural reorganization in thylakoid membranes. Biochim Biophys Acta 1817(8):1388-1391

Katoh T, Mimuro M, Takaichi S (1989) Light-harvesting particles isolated from a brown alga, Dictyota dichotoma: a supramolecular 
assembly of fucoxanthin-chlorophyll-protein complexes. Biochim Biophys Acta 976(2-3):233-240

Keller D, Bustamante C (1986) Theory of the interaction of light with large inhomogeneous molecular aggregates. II. psi-type circular dichroism. J Chem Phys 84(6):2972-2980

Larkum AW et al, Larkum M (2003) Algal plastids: their fine structure and properties. Photosynthesis in algae. Springer, Dordrecht, pp $11-28$

Larkum AW, Kühl M (2005) Chlorophyll d: the puzzle resolved. Trends Plant Sci 10(8):355-357

Lepetit B, Volke D, Szabó M, Hoffmann R, Garab G, Wilhelm C, Goss R (2007) Spectroscopic and molecular characterization of the oligomeric antenna of the diatom Phaeodactylum tricornutum. Biochemistry 46(34):9813-9822

McFadden GI (2001) Primary and secondary endosymbiosis and the origin of plastids. J Phycol 37(6):951-959

Minagawa J (2013) Dynamic reorganization of photosynthetic supercomplexes during environmental acclimation of photosynthesis. Front Plant Sci 4:513

Moreira D, Le Guyader H, Philippe H (2000) The origin of red algae and the evolution of chloroplasts. Nature 405(6782):69-72

Murakami S, Packer L (1970) Light-induced changes in the conformation and configuration of the thylakoid membrane of ulva and porphyra chloroplasts in vivo. Plant Physiol 45(3):289-299

Mustárdy L, Garab G (2003) Granum revisited. a three-dimensional model-where things fall into place. Trends Plant Sci 8(3):117-122

Nagy G, Szabó M, Ünnep R, Káli G, Miloslavina Y, Lambrev PH, Zsiros O, Porcar L, Timmins P, Rosta L (2012) Modulation of the multilamellar membrane organization and of the chiral macrodomains in the diatom Phaeodactylum tricornutum revealed by small-angle neutron scattering and circular dichroism spectroscopy. Photosynth Res 111(1-2):71-79

Nagy G, Ünnep R, Zsiros O, Tokutsu R, Takizawa K, Porcar L, Moyet L, Petroutsos D, Garab G, Finazzi G (2014) Chloroplast remodeling during state transitions in Chlamydomonas reinhardtiias revealed by noninvasive techniques in vivo. Proc Natl Acad Sci USA 111:5042-5047

Nevo R, Charuvi D, Tsabari O, Reich Z (2012) Composition, architecture and dynamics of the photosynthetic apparatus in higher plants. Plant J 70(1):157-176

Nutman AP, Bennett VC, Friend CR, Van Kranendonk MJ, Chivas AR (2016) Rapid emergence of life shown by discovery of 3700-million-year-old microbial structures. Nature 537(7621):535-538

Palmer JD, Stein DB (1986) Conservation of chloroplast genome structure among vascular plants. Curr Genet 10(11):823-833

Patty CHL, ten Kate IL, Sparks WB, Snik F (2018) Remote sensing of homochirality: a proxy for the detection of extraterrestrial life. In: Polavarapu P (ed) Chiral analysis, second edition: advances in spectroscopy, chromatography and emerging methods. Elsevier, New York, pp 29-69

Patty CHL, Luo DA, Snik F, Ariese F, Buma WJ, ten Kate IL, van Spanning RJM, Sparks WB, Germer TA, Garab G, Kudenov MW (2018) Imaging linear and circular polarization features in leaves with complete Mueller matrix polarimetry. Biochim Biophys Acta 1862(6): 1350-1363

Patty CHL, Visser LJJ, Ariese F, Buma WJ, Sparks WB, van Spanning RJM, Röling WFM, Snik F (2017) Circular spectropolarimetric sensing of chiral photosystems in decaying leaves. J Quant Spectrosc Radiat Transfer 189:303-311

Planavsky N, Asael A, Hofmann A, Reinhard C, Lalonde S, Knudsen A, Wang X, Ossa F, Pecoits E, Smith A (2014) Evidence for oxygenic photosynthesis half a billion years before the great oxidation event. Nat Geosci 10:283-286

Ponce-Toledo RI, Deschamps P, López-García P, Zivanovic Y, Benzerara K, Moreira D (2017) An early-branching freshwater cyanobacterium at the origin of plastids. Curr Biol 27(3):386-391
Pospergelis M (1969) Spectroscopic measurements of the four stokes parameters for light scattered by natural objects. Sov Astron 12:973

Premvardhan L, Robert B, Beer A, Büchel C (2010) Pigment organization in fucoxanthin chlorophyll a/c2 proteins ( $\mathrm{fcp}$ ) based on resonance raman spectroscopy and sequence analysis. Biochim Biophys Acta 1797(9):1647-1656

Remias D, Lütz-Meindl U, Lütz C (2005) Photosynthesis, pigments and ultrastructure of the alpine snow alga Chlamydomonas nivalis. Eur J Phycol 40(3):259-268

Schlodder E, Shubin VV, El-Mohsnawy E, Roegner M, Karapetyan NV (2007) Steady-state and transient polarized absorption spectroscopy of photosytem i complexes from the cyanobacteria arthrospira platensis and thermosynechococcus elongatus. Biochim Biophys Acta 1767(6):732-741

Schwieterman EW, Kiang KY, Parenteau MN, Harman CE, DasSarma S, Fisher TM, Arney GN, Hartnett HE, Reinhard CT, Olson SL, Meadows VS, Cockell CS, Walker SI, Grenfell JL, Hegde S, Rugheimer S, Hu R, Lyons TW (2018) Exoplanet biosignatures: a review of remotely detectable signs of life. Astrobiology 18(6):663-708

Sparks WB, Hough J, Germer TA, Chen F, DasSarma S, DasSarma P, Robb FT, Manset N, Kolokolova L, Reid N (2009) Detection of circular polarization in light scattered from photosynthetic microbes. Proce Natl Acad Sci USA 106(19):7816-7821

Sparks W, Hough J, Kolokolova L, Germer T, Chen F, DasSarma S, DasSarma P, Robb F, Manset N, Reid I, Macchetto F, Martin W (2009) Circular polarization in scattered light as a possible biomarker. J Quant Spectrosc Radiat Transfer 110(14-16):1771-1779

Staehelin L (1986) Chloroplast structure and supramolecular organization of photosynthetic membranes. Photosynthesis III. Springer, New York, pp 1-84

Steinbach G, Pawlak K, Pomozi I, Tóth EA, Molnár A, Matkó J, Garab G (2014) Mapping microscopic order in plant and mammalian cells and tissues: novel differential polarization attachment for new generation confocal microscopes (DP-LSM). Methods Appl Fluoresc 2(1):015005

Steinmann E, Sjöstrand F (1955) The ultrastructure of chloroplasts. Expe Cell Res 8(1):15-23

Szabó M, Lepetit B, Goss R, Wilhelm C, Mustárdy L, Garab G (2008) Structurally flexible macro-organization of the pigment-protein complexes of the diatom Phaeodactylum tricornutum. Photosynth Res 95(2-3):237-245

Tinoco I Jr, Mickols W, Maestre M, Bustamante C (1987) Absorption, scattering, and imaging of biomolecular structures with polarized light. Annu Rev Biophys Biophys Chem 16(1):319-349

Tokutsu R, Kato N, Bui KH, Ishikawa T, Minagawa J (2012) Revisiting the supramolecular organization of photosystem II in Chlamydomonas reinhardtii. J Biol Chem 287(37):31574-31581

Trissl H-W, Wilhelm C (1993) Why do thylakoid membranes from higher plants form grana stacks? Trends Biochem Sci 18(11):415-419

Tóth TN, Rai N, Solymosi K, Zsiros O, Schröder WP, Garab G, van Amerongen H, Horton P, Kovács L (2016) Fingerprinting the macro-organisation of pigment-protein complexes in plant thylakoid membranes in vivo by circular-dichroism spectroscopy. Biochim Biophys Acta 1857(9):1479-1489

Wellman CH, Gray J (2000) The microfossil record of early land plants. Philos Trans R Soc Lond B 355(1398):717-732

Wolstencroft RD (1974) The circular polarization of light reflected from certain optically active surfaces. In: IAU Colloq. 23: Planets, Stars, and Nebulae: Studied with Photopolarimetry, p. 495 